\title{
Estimation of normalized point-source sensitivity of segment surface specifications for extremely large telescopes
}

\author{
Byoung-Joon Seo,,$^{1,2, *}$ Carl Nissly, ${ }^{1,2}$ Mitchell Troy, ${ }^{1,2}$ George Angeli, ${ }^{3}$ \\ Robert Bernier, ${ }^{3}$ Larry Stepp, ${ }^{3}$ and Eric Williams ${ }^{3}$ \\ 'Jet Propulsion Laboratory, Pasadena, California 91109, USA \\ ${ }^{2}$ California Institute of Technology, Pasadena, California 91125, USA \\ ${ }^{3}$ Thirty Meter Telescope Observatory, Pasadena, California 91107, USA \\ ${ }^{*}$ Corresponding author: Byoung-Joon.Seo@ jpl.nasa.gov
}

Received 18 December 2012; revised 25 February 2013; accepted 25 February 2013; posted 1 May 2013 (Doc. ID 181977); published 12 June 2013

\begin{abstract}
We present a method which estimates the normalized point-source sensitivity (PSSN) of a segmented telescope when only information from a single segment surface is known. The estimation principle is based on a statistical approach with an assumption that all segment surfaces have the same power spectral density (PSD) as the given segment surface. As presented in this paper, the PSSN based on this statistical approach represents a worst-case scenario among statistical random realizations of telescopes when all segment surfaces have the same PSD. Therefore, this method, which we call the vendor table, is expected to be useful for individual segment specification such as the segment polishing specification. The specification based on the vendor table can be directly related to a science metric such as PSSN and provides the mirror vendors significant flexibility by specifying a single overall PSSN value for them to meet. We build a vendor table for the Thirty Meter Telescope (TMT) and test it using multiple mirror samples from various mirror vendors to prove its practical utility. Accordingly, TMT has a plan to adopt this vendor table for its M1 segment final mirror polishing requirement. (C) 2013 Optical Society of America
\end{abstract}

OCIS codes: (110.6770) Telescopes; (110.3925) Metrics.

http://dx.doi.org/10.1364/AO.52.004111

\section{Introduction}

The use of segmented mirrors is a key innovation of many modern large-aperture telescopes. Although this segmented approach surely avoids the difficulty of fabricating one large monolithic mirror, the complexity added by multiple segments needs to be controlled in order to utilize their scalability properly. These segments have to be well polished and aligned. Their surfaces need to be maintained by supporting structures such as warping harnesses (WHs) for surface shape compensation. Therefore, overall

$1559-128 \mathrm{X} / 13 / 184111-12 \$ 15.00 / 0$

(C) 2013 Optical Society of America performance of a segmented telescope depends on the ensemble behavior of all segments including their fabrication, alignment, operation, and control strategy.

These considerations make it challenging to develop an individual specification for segments, such as the segment polishing specification. The segment polishing specification is applicable to a single segment while it should be made to meet the overall telescope science requirement when all segments satisfy the specification. It should also consider the telescope's control and alignment capability. At the same time, it should be simple and straightforward enough for the mirror vendors, encapsulating its complexity; that is, the mirror polishing vendors 
do not need to understand the complex science requirement of the telescope.

In this paper, we introduce the vendor table, which approximates the normalized point-source sensitivity (PSSN) of a fully populated segmented telescope from a single segment surface's polishing information. The PSSN is a recently developed performance metric that has been adopted for the Thirty Meter Telescope (TMT) and European Extremely Large Telescope for their error budgeting and performance evaluation [1-4]. This metric is directly related to the photometric error of background-limited observations so that it accurately represents the efficiency loss of science due to increased telescope observing time $[\underline{5}, \underline{6}]$.

In principle, the PSSN, or the overall telescope performance in general, cannot be estimated from only a single segment's surface information. It requires the polishing information of all segments and also their surface control scheme to estimate the overall telescope performance metrics. Therefore, the PSSN estimation from a single segment can be used only for testing purposes to determine whether the surface quality of the test sample mirror meets the science requirement of the overall telescope performance or not. In this case, the single segment will be referred to as the mirror under test (MUT).

One straightforward way of estimating a PSSN from a MUT is to consider an imaginary telescope, which has identically replicated segments as the MUT. In this imaginary replicated telescope, all segments are assumed to be polished the same, installed in the same direction, and aligned and controlled in the same manner. However, this imaginary replicated telescope is not likely to be built in practice. If all segments are polished from the same set of machines and processes of the same vendor, it is more probable that the residual surface errors after polishing have some deterministic errors and some random errors. The deterministic errors are the same error observed in all segments due to static defects in the polishing process while the random errors are unique errors from segment to segment due to the randomness of the polishing process. It is also likely that the random errors follow certain statistics, which will be dependent on the specific polishing process.

The vendor table assumes that the polishing errors observed from the MUT are all random errors, and all other segments have the same amount of aberration for each aberration frequency as the MUT. In other words, it assumes that all other segments have the same power spectral density (PSD) of the surface aberration as that of the MUT. As presented later in Sections $\underline{3}$ and $\underline{5}$, the PSSN with a random segment configuration is worse than comparing to that with the homogeneous or replicated segment configuration. Thus, the estimated PSSN using this vendor table represents a worst-case scenario of the telescope configuration when all segments have random errors yet having the same PSD as the MUT. In this case, it is safe to say that the overall telescope's performance meets the PSSN requirement if the PSSN of each segment estimated by this vendor table is better than the overall PSSN requirement. This feature makes the vendor table useful for individual segment specification such as the segment polishing specification. Therefore, it is currently TMT's plan to adopt this vendor table approach for its M1 segment final mirror polishing specification [7]. A specification based on the vendor table can be directly related to the telescope science metric, such as PSSN, and provides the mirror vendors flexibility in how to polish by specifying a single PSSN value for them to meet.

As presented later in Section 2, the vendor table is based on the parametric decomposition of the MUT surface. One might regard this as a somewhat complicated process of estimating a PSSN since there are simpler approximate methods such as $\beta$ approximation and Slope RMS approximation [8]. However, we find that none of these approximation methods is applicable for the complex M1 system. The Slope RMS approximation works only for low-spatialfrequency aberration, while the spatial frequency for the conventional M1 segment surfaces is higher than its applicable frequency limit, namely, the breaking frequency. Applying the $\beta$ approximation is also challenging since the accurate estimate of a MUT's surface PSD is difficult after considering the telescope's control. The $\beta$ approximation can introduce additional practical problems as discussed in Section 4.B.

This paper is organized as follows. In Section 2, we first describe the vendor table itself, showing how one can estimate a PSSN using it. We also present a vendor table that we have developed for the TMT as an example. In Section 3, we discuss the philosophical background that validates the statistical approach on which the vendor table is based. We prove that the PSSN with a random segment configuration is worse than that with the homogeneous or replicated segment configuration. In Section 4, we present our method to derive the sensitivity coefficients for the TMT vendor table. Then, we show the validation of this TMT vendor table in Section 5. Lastly, in Section $\underline{6}$, we summarize by discussing the usefulness and the limitations of the vendor table.

\section{Vendor Table}

Table 1 shows an example vendor table, which is implemented for TMT M1 segments. As discussed, the vendor table approximates PSSN from the single segment surface information of the MUT. We follow the steps below in Table $\underline{1}$ to estimate the PSSN.

A. We first decompose the MUT into a parameter space consisting of low-order error, high-order residual, and surface roughness error. The low-order errors in Table 1 are represented by the first 15 Zernike modes [9], that is, 15 low-order Zernikes (LOZs), which are specifically used for TMT. The first 15 LOZs measured from the MUT are located under the $Z_{M}$ column. The values shown in Table 1 are the Zernike decomposition values from one of our 


\begin{tabular}{|c|c|c|c|c|c|}
\hline \multicolumn{4}{|c|}{ Surface } & & $\begin{array}{r}\text { PSSN Estimate } \\
0.96237\end{array}$ \\
\hline Low order Zernike & $\begin{array}{c}Z_{M} \\
{\left[n m_{R M S}\right]}\end{array}$ & $\begin{array}{c}Z_{E} \\
{\left[n m_{R M S}\right]}\end{array}$ & $\begin{array}{c}\mathrm{LOZ} \\
{\left[n m_{R M S}\right]}\end{array}$ & $\begin{array}{c}\alpha^{\prime} \\
{\left[1 / n m^{2}\right]}\end{array}$ & 0.98809 \\
\hline Focus $\left(Z_{2,0}\right)$ & 4.8 & 50.0 & 50.2 & $1.1165 \times 10^{-6}$ & 0.99718 \\
\hline $\operatorname{Astigmatism}\left(Z_{2,+2}\right)$ & 2.1 & 70.7 & 70.7 & $3.7670 \times 10^{-7}$ & 0.99812 \\
\hline Astigmatism $\left(Z_{2,-2}\right)$ & -0.2 & 70.7 & 70.7 & $3.7619 \times 10^{-7}$ & 0.99812 \\
\hline Coma $\left(Z_{3,+1}\right)$ & 0.0 & 7.1 & 7.1 & $3.0264 \times 10^{-5}$ & 0.99847 \\
\hline $\operatorname{Coma}\left(Z_{3,-1}\right)$ & -0.1 & 7.1 & 7.1 & $3.0611 \times 10^{-5}$ & 0.99846 \\
\hline Trefoil $\left(Z_{3,+3}\right)$ & 0.7 & 14.1 & 14.1 & $3.2339 \times 10^{-6}$ & 0.99936 \\
\hline Trefoil $\left(Z_{3,-3}\right)$ & 0.8 & 14.1 & 14.1 & $5.7444 \times 10^{-7}$ & 0.99989 \\
\hline Spherical $\left(Z_{4,0}\right)$ & -4.5 & 0.0 & 4.5 & $6.0662 \times 10^{-5}$ & 0.99877 \\
\hline Secondary astigmatism $\left(Z_{4,+2}\right)$ & 0.8 & 0.0 & 0.8 & $1.4037 \times 10^{-4}$ & 0.99991 \\
\hline Secondary astigmatism $\left(Z_{4,-2}\right)$ & -1.3 & 0.0 & 1.3 & $1.3926 \times 10^{-4}$ & 0.99976 \\
\hline Quadrafoil $\left(Z_{4,+4}\right)$ & 0.5 & 0.0 & 0.5 & $1.0893 \times 10^{-5}$ & 1.00000 \\
\hline Quadrafoil $\left(Z_{4,-4}\right)$ & -0.2 & 0.0 & 0.2 & $1.0804 \times 10^{-5}$ & 1.00000 \\
\hline & & & $\begin{array}{c}r m s S R F_{H O} \\
{\left[n m_{R M S}\right]}\end{array}$ & $\begin{array}{c}\alpha_{H O} \\
{\left[1 / \mathrm{nm}^{2}\right]}\end{array}$ & \\
\hline High order residual & & & 5.8 & $7.7380 \times 10^{-4}$ & 0.97397 \\
\hline & & & $\begin{array}{c}r m s S R F_{S R} \\
{\left[n m_{R M S}\right]}\end{array}$ & $\begin{array}{c}\alpha_{S R} \\
{\left[1 / \mathrm{nm}^{2}\right]}\end{array}$ & \\
\hline Surface roughness & & & 0.0 & $1.2633 \times 10^{-3}$ & 1.00000 \\
\hline
\end{tabular}

${ }^{a}$ See text for detailed explanation.

segment samples, namely Vendor-B. The computed PSD for the Vendor-B sample mirror surface is shown later in Fig. 5. Its physical characteristics are also summarized in Table 2 .

Table 2. Real Mirror Samples Used in This Paper ${ }^{a}$

\begin{tabular}{|c|c|c|c|c|c|}
\hline Mirror & $\begin{array}{l}\text { Grid } \\
\text { Size } \\
{[\mathrm{mm}]}\end{array}$ & $\begin{array}{c}\text { Max } \\
\text { Size } \\
{[\mathrm{mm}]}\end{array}$ & $\begin{array}{c}\mathrm{rmsSRF}^{b} \\
{[\mathrm{~nm}]}\end{array}$ & $\begin{array}{c}\mathrm{rmsSRF}^{c} \\
{[\mathrm{~nm}]}\end{array}$ & Comments \\
\hline Gemini & 32.00 & 8100.0 & 15.7 & 15.7 & $\begin{array}{l}\text { Circular with inner } \\
\text { circular obscuration, } \\
15 \text { Zernikes are } \\
\text { already removed in } \\
\text { original data }\end{array}$ \\
\hline Vendor-A & 5.00 & 1430.0 & 407.1 & 163.0 & Hexagonal \\
\hline Vendor-B & 4.80 & 1434.5 & 9.5 & 5.8 & $\begin{array}{l}\text { Original data is } \\
\text { hexagonal. } \\
\text { Truncated to circular } \\
\text { stated due to bad } \\
\text { edge quality }\end{array}$ \\
\hline Vendor-C & 3.20 & 1400.8 & 5.3 & 2.8 & Circular \\
\hline Vendor-D & 0.52 & 453.6 & 14.0 & 12.7 & $\begin{array}{l}\text { Circular with inner } \\
\text { circular obscuration }\end{array}$ \\
\hline Vendor-E & 2.00 & 1398.0 & 25.3 & 3.8 & Hexagonal \\
\hline Vendor-E.1 & 2.00 & 1402.0 & 23.3 & 4.5 & Hexagonal \\
\hline Vendor-E.2 & 2.00 & 1402.0 & 52.5 & 12.5 & Hexagonal \\
\hline Vendor-E.4 & 2.00 & 1406.0 & 38.9 & 11.5 & Hexagonal \\
\hline Vendor-H.1 & 0.59 & 549.4 & 16.0 & 10.4 & Circular \\
\hline Vendor-H.2 & 1.00 & 447.9 & 10.8 & 6.8 & Circular \\
\hline
\end{tabular}

${ }^{a}$ We conceal some of the vendor names per the vendors' requests. Their PSD computation is summarized in Fig. 5.

${ }^{b}$ Mirror surface before $15 \mathrm{LOZ}$ removed.

${ }^{c}$ Mirror surface after $15 \mathrm{LOZ}$ removed.
B. We can also consider the estimation uncertainty for each low-order error term (or LOZ for TMT). These values are located under the $Z_{E}$ column. Here the telescope project can specify the maximum allowable measurement uncertainty for each term. TMT refers to these terms as "LOZ allowances." The values shown in Table 1 correspond to the allocation given by the TMT Mī requirement values [10].

C. We assume that $Z_{M}$ and $Z_{E}$ are statistically independent. The values under the "LOZ" column represent the root-square-sum of $Z_{M}$ and $Z_{E}$ values.

$D$. We define the high-order residual as the residual surface error after the low-order error is removed, and we define the surface roughness error for even higherfrequency aberration than the high-order residual. More detail on these frequency boundaries is described in Section 4.

Then, the RMS surface error (rmsSRF) of the highorder residual and the surface roughness error are measured and located right next to the high-order residual and surface roughness error row. The high-order residual value shown in Table 1 is the measured value from the same segment sample, Vendor-B, after removing the first 15 LOZs. The surface roughness error value in Table $\underline{1}$ is assigned 0 due to the lack of input information.

E. For each term, we obtain its sensitivity values. They are alpha prime coefficients for low-order error $\alpha_{i}^{\prime}$ (Zernike for this example), alpha coefficients for high-order residual $\alpha_{H O}$, and alpha coefficients for surface roughness error $\alpha_{S R}$. These $\alpha$ coefficients represent the PSSN sensitivity from the rmsSRF of 
each term to their corresponding PSSN values. They are precalculated and system-dependent values, which take the telescope controls into account such as the segment piston, tip-tilt alignment, and $\mathrm{WH}$ correction.

The $\alpha$ coefficients in Table 1 are computed based on WH influence functions of the TMT segment as described in Section 4 . Using the $\alpha$ coefficients, we estimate the PSSN contribution from each term as follows:

$$
\left\{\begin{array}{l}
\operatorname{PSSN}_{L O}=\prod_{i=4}^{15}\left(1-\alpha_{i}^{\prime} \cdot Z_{i}^{2}\right) \\
\operatorname{PSSN}_{H O}=1-\alpha_{H O} \cdot r m s S R F_{H O}^{2} \\
\operatorname{PSSN}_{S R}=1-\alpha_{S R} \cdot r m s S R F_{S R}^{2}
\end{array},\right.
$$

where $r m s S R F_{H O}$ and $r m s S R F_{S R}$ are the rmsSRF of the high-order residual and the surface roughness error, respectively.

F. Finally, we estimate the overall PSSN by multiplying each PSSN contribution as shown in the upper right corner in the PSSN box:

$$
P S S N=P S S N_{L O} \times P S S N_{H O} \times P S S N_{S R}
$$

Therefore, the overall PSSN estimation is based on the multiplicative feature of PSSN [6]. A similar technique can be used for other science metrics. We have also analyzed the adaptive optics corrected rms wavefront error (rmsWFE) and WH stroke usage for TMT segments [7].

\section{Validation of Statistical Approach}

First, let us imagine a segmented telescope, which has a diffraction-limited point spread function (PSF) located only at the center pixel of a CCD-type detector array. Then, suppose the following imaginary aberration case: (1) All segments have exactly the same surface aberration, (2) all segments are arranged in a homogeneous way or orientation, and (3) the segment aberration makes an optical path difference (OPD), which pushes the center PSF energy to a certain pixel away with a fraction of $\delta$. The left part of Fig. 1 demonstrates this situation.

The corresponding PSSN of the homogeneously arranged telescope is computed as follows:

$$
\operatorname{PSSN}_{H}=(1-\delta)^{2}+\delta^{2} .
$$

Now suppose that the segments are rearranged randomly. Then, the energy will spread evenly in all directions as depicted in the right part of Fig. 1 . Then, the corresponding PSSN of the randomly arranged telescope is computed as

$$
\operatorname{PSSN}_{R}=(1-\delta)^{2}+\frac{\delta^{2}}{2} .
$$

We find that the PSSN of the homogeneously arranged telescope is larger than that of the randomly arranged telescope:

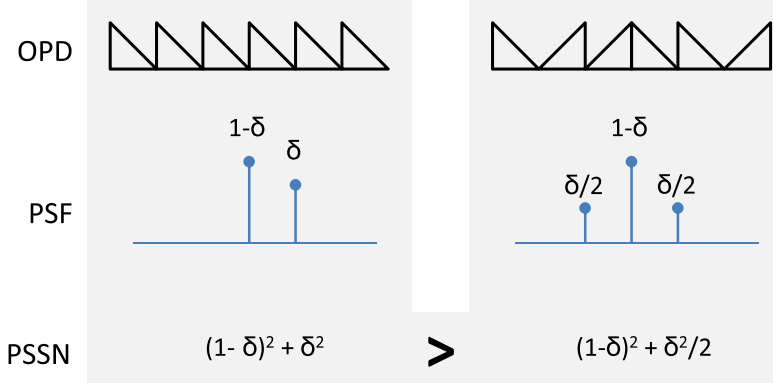

Fig. 1. Two differently configured telescopes are compared conceptually. Left is for a telescope where segments are arranged homogeneously. Right is for a telescope where segments are arranged randomly. Both telescopes have a single segment type, whose anisotropic OPD aberration makes the PSF spread to a point away from its center core in only one direction. This imaginary conceptual situation is only for demonstrating the correctness of Eq. (5).

$$
P S S N_{H} \geq P S S N_{R}
$$

Note that there will not be such an aberration as discussed here in a real telescope due to the Fourier transform property; that is, no single pixel PSF or no surface aberration which introduces a single frequency shift. We use this imaginary aberration only for conceptual demonstration purposes to describe Eq. (5). However, this can be proved to be generally true for any nonisotropic segment aberration. $P S S N_{H}$ becomes equal to $P S S N_{R}$ if the aberration from a single segment is isotropic.

Figure 2 shows the similar but more realistic situation in two-dimensional (2D) space. The following assumptions are made in Fig. 2: (1) The telescope has four identical rectangular segments and (2) the segment has a sinusoidal aberration along its diagonal direction with the spatial frequency $2.2 \times \sqrt{2} / \mathrm{m}$, and rmsWFE of $14.1 \mathrm{~nm}$.

We consider two configurations in terms of segment arrangements. (1) Figure 2(a) arranges the segments homogeneously and (2) Fig. 2(b) arranges the segments in a zig-zag pattern. The corresponding PSFs are shown in Figs. 2(c) and 2(d). Similar to the discussion for Fig. 1, the PSF is spread in more diverse directions for Fig. 2(d). Consequently, the corresponding PSSN is larger in Fig. 2(c) than that in Fig. 2(d).

We can generalize the result as follows. First, between two telescopes which have all of the same segments but arranged differently, the PSSN of the more randomly arranged telescope is worse. However, in a real situation, all segments cannot be identical. If all segments are manufactured through the same mechanical and optical processes, it is more likely that the residual surface error has some deterministic errors and some random errors. The deterministic errors are the same error observed in all segments while the random errors are unique for each segment. It is also likely that the random errors follow certain statistics, which will be dependent on the specific polishing process. 


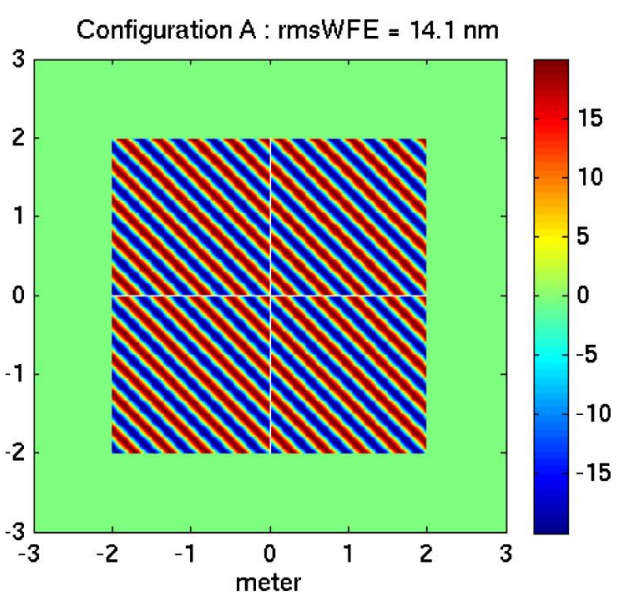

(a)

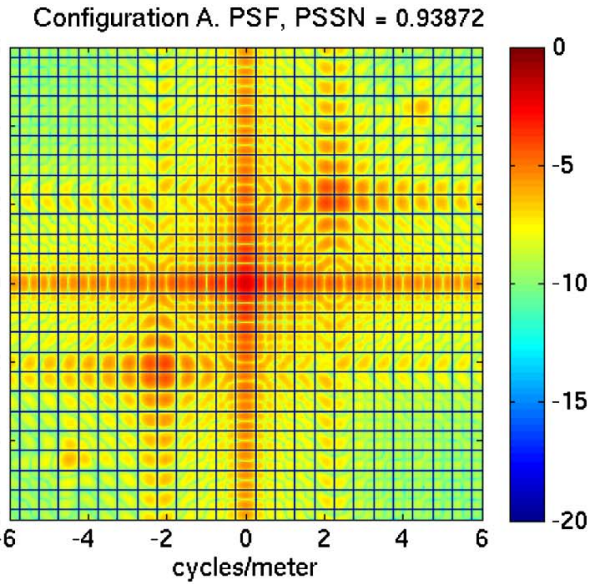

(c)

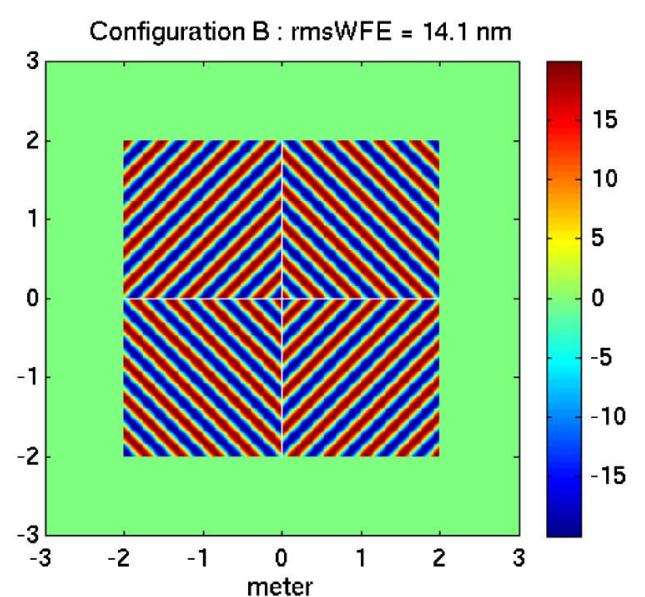

(b)

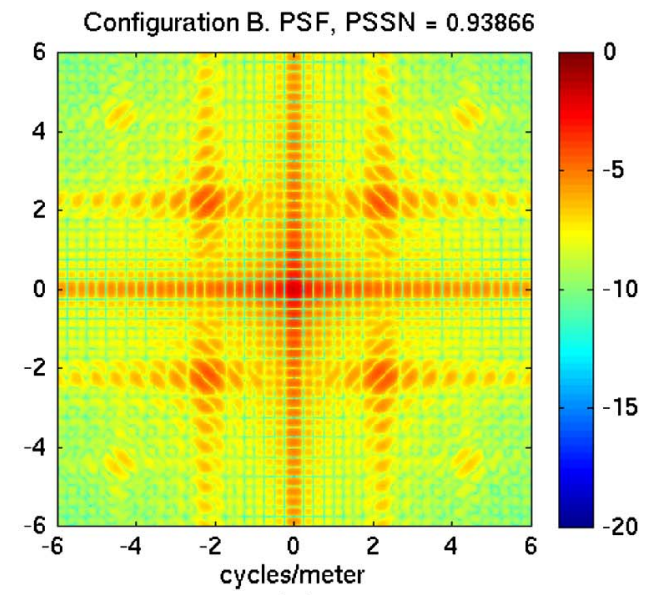

(d)

Fig. 2. Two differently configured telescopes are compared as the $2 \mathrm{D}$ version of Fig. 1 . (a) is for a telescope that arranges the segments homogeneously while (b) is for the same telescope but with segments arranged in a zig-zag pattern. The corresponding PSFs are shown in (c) and (d), respectively. Similarly as in the discussion for Fig. 1, the PSF is spread in more diverse directions in (d). Consequently, the corresponding PSSN is larger in (c) than that in (d). This conceptual situation is for demonstrating the correctness of Eq. (5).

If we identify those deterministic errors and the statistics of the random errors, it becomes possible to build an arbitrarily synthesized telescope, which represents one possible random realization of a realworld telescope. Then, the PSSN of this arbitrarily synthesized telescope will be smaller than the PSSN of the replicated telescope.

Nonetheless, it is often challenging to identify those deterministic errors and statistics of the random errors. Instead of finding these, we can make an even more aggressive assumption that all polishing errors observed in the test sample segment are random errors and all the other segments have the same PSD as the MUT. Then the PSSN based on the same PSD will be even smaller than the PSSNs of the other two above scenarios.

The vendor table estimates the latter case, and this forms the conceptual background for our vendor table approach, which estimates a PSSN for the most random telescope, and therefore the worst-case PSSN. In Section $\underline{5}$, we further verify this by using real mirror segment samples.

\section{Vendor Table Sensitivities}

As shown in Table 1, we decompose the MUT into three subgroups: low-order error, high-order residual, and surface roughness error.

The low-order error is defined as the surface aberration, which can be influenced by the telescope's control, that is, $\mathrm{WH}$ and piston-tip-tilt control. The high-order residual is defined as the remainder beyond the low-order error, which is typically measured by the center-of-curvature interferometry test. The surface roughness error is defined as the surface aberration whose spatial frequency is even higher than this high-order residual. The surface roughness is typically measured or estimated in an ultrasubaperture level or in a line-scanning mode using a special tool such as a Chapman profilometer.

There can be an uncovered frequency gap between the high end of the high-order residual and the low end of the surface roughness error due to practical limitations of those measurement systems. The typical frequency range of the gap is between $1 \mathrm{~mm}^{-1}$ and $100 \mu \mathrm{m}^{-1}$. However, we find that the surface errors in 
this gap is negligibly small for most reasonable mirrors after inspecting our mirror samples in Fig. 5. We assume that their PSDs behave similarly beyond their sampling permitted measurable frequency. Therefore, we ignore the impact from this possible missing frequency gap when evaluating the vendor table.

For the TMT example in Table 1, we consider the first 15 Noll Zernike modes [9] $(15 \mathrm{LOZ})$ defined within MUT for the low-order error. We also use $1 / 100 \mu \mathrm{m}^{-1}$ for the frequency separation bound between the high-order residual and the surface roughness error. The $1 / 100 \mu \mathrm{m}^{-1}$ is chosen based on the smallest frequency band that a Chapman profilometer can measure.

\section{A. Low-Order Error}

Both the choice of the basis function set for the loworder error and the number of the basis functions are system-dependent. Instead of LOZ, other kinds of basis function sets can be used. One can consider the hexikies, for example, which form an orthogonal basis on the hexagonal segment. Another choice for the basis function set is the singular value decomposition (SVD) modes of the WH influence functions. The SVD modes form an orthogonal basis for system-dependent segment shape, and they will eliminate the need for the sensitivity coefficients because such a mode will be completely corrected by the WH by definition.

We choose Zernikes for TMT based on the following reasons. First, Zernikes are universal and widely used by mirror vendors, which makes it easy to communicate with mirror vendors avoiding possible misunderstanding. Second, Zernikes are well aligned with optics naturally. In particular, optics shop test errors (random and systematic) due to misalignment show up as LOZs. If we can employ the SVD modes instead of Zernikes, the projections of these natural Zernike-type errors corrected by the SVD modes have to be still considered in the vendor table. For example, $80 \%$ of coma can be corrected by the WH influence functions. (Coma can be expanded into SVD modes with $20 \%$ residual error.) In the physical sense, this becomes the same limitation as the choice of Zernikes.

Once a set of basis functions is defined for the low-order error, we evaluate the corresponding sensitivity coefficients from each term to PSSN. Since the low-order error is corrected by the telescope's controls, we need to consider their effects for the coefficient evaluation.

Unless the SVD modes are used for the basis function set for the low-order error, any Zernike mode, as an example of the basis functions, leaves a certain amount of residual after WH control. Figure 3 shows the rmsSRF ratio before and after WH correction for each Zernike mode. The green and red curves are for 10 or 21 SVD mode correction. The TMT WH for the M1 segment has 21 influence functions, thus 21 SVD modes total although the TMT's baseline (as

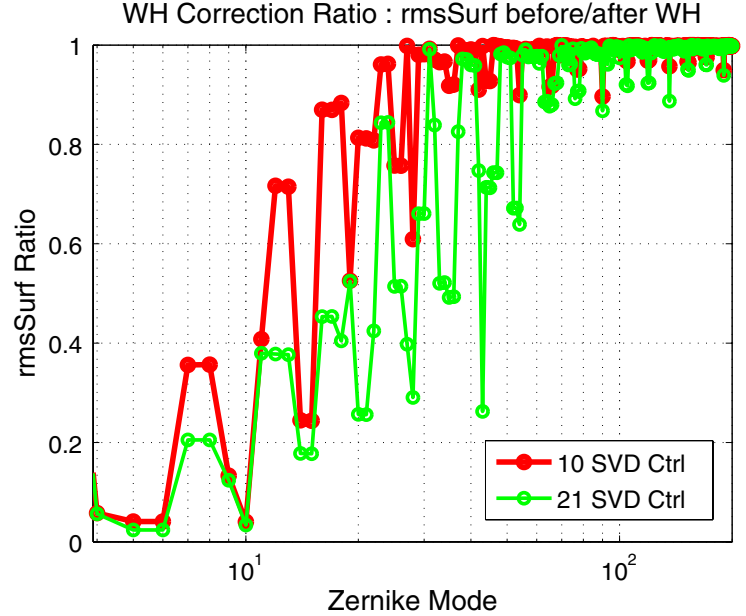

Fig. 3. rmsSRF ratio by WH correction for each Zernike mode. The green and red curves are for 10 or 21 SVD mode correction. TMT WH for M1 segment has 21 influence functions, and thus 21 SVD modes total.

of December 2012) is to only correct using 10 SVD modes.

The alpha prime coefficient $\alpha_{i}^{\prime}$ relates the sensitivity between the residual of each Zernike and the corresponding PSSN. In order to obtain $\alpha_{i}^{\prime}$, we have performed the analysis described in Fig. $\underline{4}$ and as follows.

1. We define Zernike coefficients, $Z_{i}$, on the segment circumscribed aperture (not hexagonal) with diameter of $1.44 \mathrm{~m}$. Then, the hexagonal shape is extracted to make a segment.

2. We apply WH correction [11] to the segment. 10 SVD mode control is applied as a baseline. We perform additional piston, tip, and tilt removal afterward.

3 . We repeat the above process to generate 492 segments to form a set of TMT M1. The same number of Zernike coefficients are applied to all segments. However, we assume random polarity or phase (segment installation direction) of the Zernikes on each segment as shown in Fig. 4.

4. We numerically install this full set of TMT M1 segments in our ray-tracing modeling tool, modeling and analysis for controlled optical systems (MACOS), from which we obtain the simulated wavefront OPD. Then, we compute PSSN using the OPD [6]. We repeat the above processes multiple times (Monte Carlo) to get averaged values of PSSN, $\widehat{P S S N}$. The PSSN variation is due to the random sign polarity, and the rmsWFE variation is negligible.

5. Obtain the alpha prime coefficients using the following equation:

$$
\alpha_{i}^{\prime}=\frac{1-\widehat{P S S N}}{Z_{i}^{2}} .
$$

We chose 15 Zernike modes for the LOZ of TMT after we consider the overall estimation accuracy of the vendor table including the high-order residual, which is described in detail in Section 4.B. 


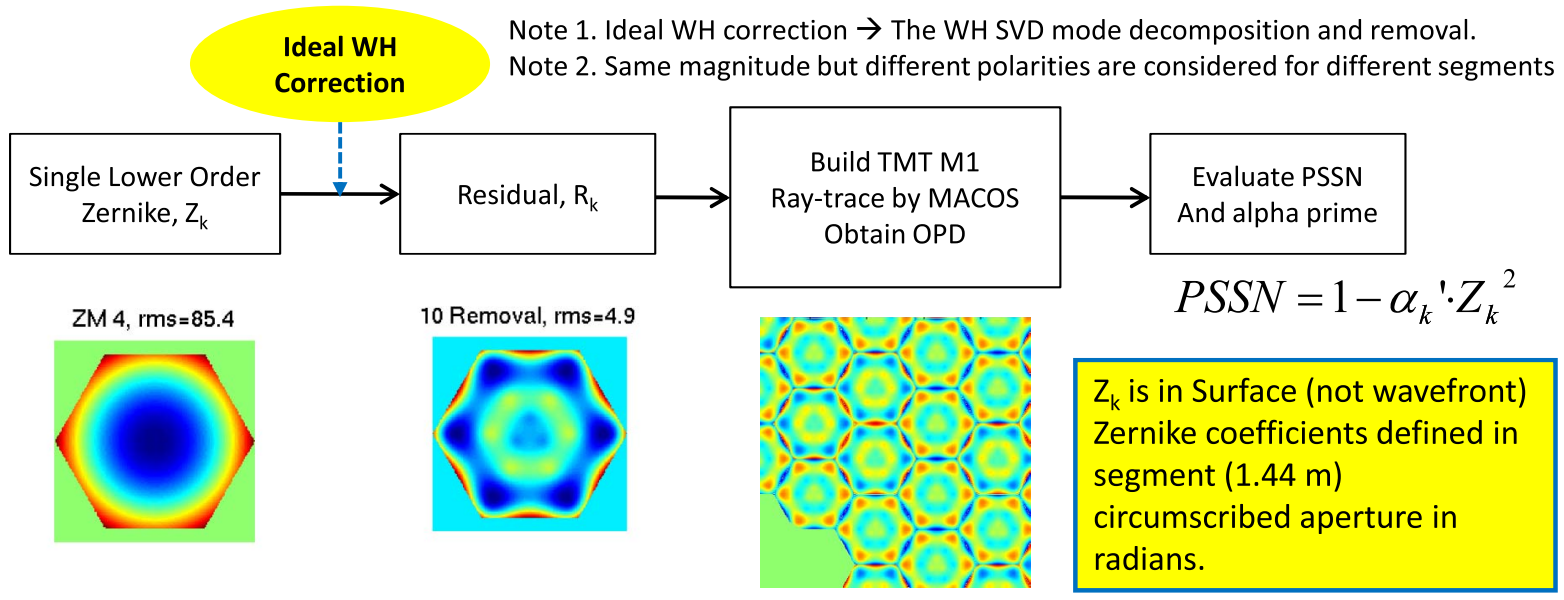

Fig. 4. Procedure to compute the alpha prime $\alpha^{\prime}$ coefficients. The segment focus mode is used as an example.

\section{B. High-Order Residual Error}

In order to estimate the PSSN corresponding to the high-order residual, it would be best to find the PSD of the MUT in spatial frequency space. With the computed PSD, one can use the $\beta$ approximation [8] to estimate its PSSN. The $\beta$ approximation is a PSSN approximation using the PSD and the $\beta$ function, which is the spatial frequency-dependent sensitivity to PSSN. The $\beta$ approximation assumes that each frequency component contributes to the overall PSSN independently.

However, this method would introduce many practical problems if the PSD computation step is required in the vendor table, especially when it is meant for the utility of polishing specification. All of the detailed computation steps have to be defined and documented by the party who builds the specification while they should be well understood and executed by the mirror vendors. Such a complex specification will generate technical ambiguity and misunderstanding between the two parties.

In order to maintain a simple specification, we propose to use a single value for describing the highorder residual: rmsSRF for the high-order residual

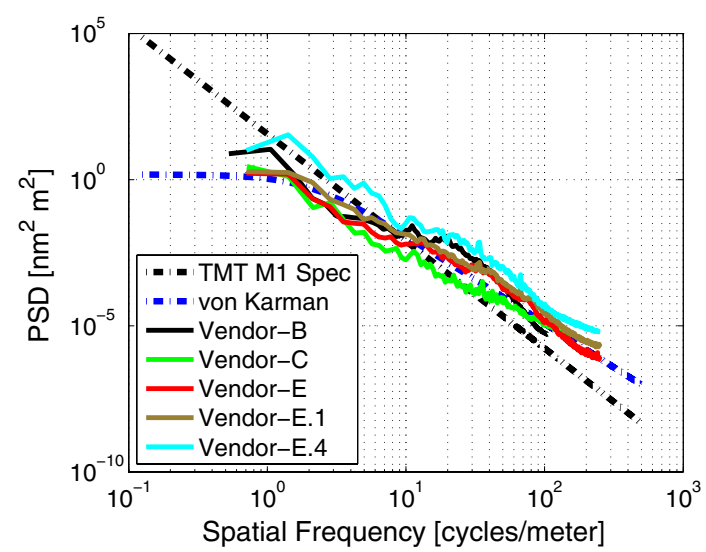

(a)Group 1 denoted by $r m s S R F_{H O}$. The immediate trade-off for this simplification is the difficulty in computing the sensitivity coefficient $\left(\alpha_{H O}\right)$ from $r m s S R F_{H O}$ to its corresponding PSSN. According to our definition of the high-order residual described earlier in this section, it is not always clear where the low-frequency bound for the high-order residual is located in spatial frequency space. This is because of the discrepancy between WH influence functions and the sinusoidal functions. Even if the low (and high) frequency band of the high-order residual is clearly defined, the $\beta$ approximation implies that $\alpha_{H O}$ is not defined clearly unless we know the PSD of this high-order residual.

We use a value of $1.225 / \mathrm{rad}^{2}$ for $\alpha_{H O}$ for TMT. The following are the assumptions and method used to find this value. We first obtained surface measurements of real mirrors from various mirror vendors. This data is summarized in Table 2 , where we conceal some of the vendor names per the vendors' requests. We then computed their PSDs as shown in Fig. 5. The black dashed line is the PSD interpretation of the current TMT structure function specification [12], which is the surface representation of the atmosphere turbulence Kolmogorov spectrum [9]

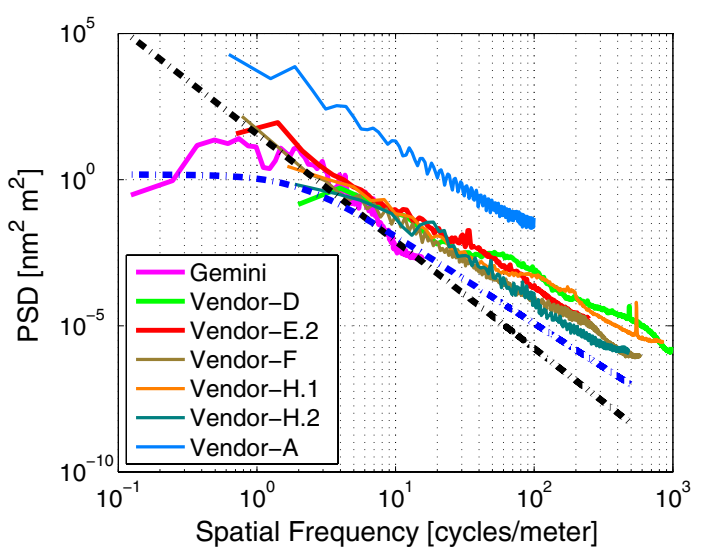

(b)Group 2

Fig. 5. PSD computed from surface measurements of real mirrors from various vendors listed in Table 2. The black dashed line and the blue dashed line in both plots are the reference PSDs, representing the PSD interpretation of the current TMT structure function specification [12] and a von Karman PSD with parameters of $A=1.5 \mathrm{~nm}^{2} \mathrm{~m}^{2}, f_{o}=2$ cycles $/ \mathrm{m}$, and $\gamma=3$. We split the data set into two groups to make plots easier to read. 


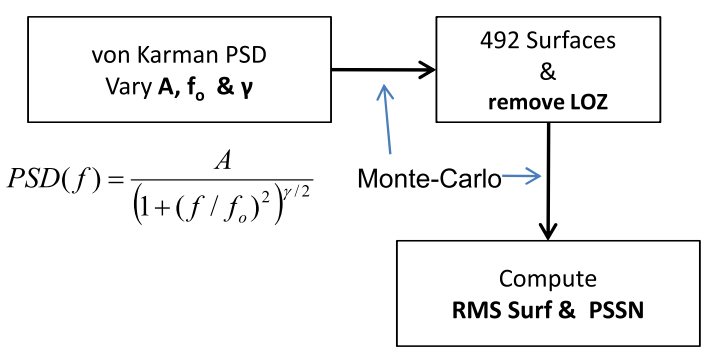

Fig. 6. Procedure to compute $\alpha_{v k}$, the alpha value based on the von Karman PSD.

with the Fried parameter $r_{o}$ of $1 \mathrm{~m}$. The von Karman PSD (its formula is in Fig. 5) is also shown in Fig. 5 in the blue dashed line with parameters of $A=1.5 \mathrm{~nm}^{2} \mathrm{~m}^{2}, f_{o}=2$ cycles $/ \mathrm{m}$, and $\gamma=3$. The parameters of $f_{o}=2$ cycles $/ \mathrm{m}$ and $\gamma=3$ are chosen for representing the sample mirrors for the $\alpha_{H O}$ evaluation later in this section.

In Fig. $\underline{5}$, we observe that the PSD slopes from all real mirror samples are close to 3 while that of the Kolmogorov PSD is 11/3. We also notice that the energy in the low frequency is finite for the real mirror samples and has a knee frequency of around 2 cycles $/ \mathrm{m}$ while the Kolmogorov PSD approaches infinity near zero frequency. We believe the knee frequency is physically related to the polishing tool size. Based on these observations, we assume that most mirrors have a PSD slope and knee frequency close to 3 and 2 cycles $/ \mathrm{m}$, respectively. In other words, the high-order residual after the low-order-error removal has a similar PSD spectrum except for the amplitude scale. We also assume that aberrations in the high-order residual regime can be treated as random errors (not deterministic), that is, all other segments have the same PSD but different phases for each frequency.

Based on these assumptions, we perform a study described below and shown in Fig. 6 . We first generate 492 random surfaces for TMT M1 segments using the von Karman PSD. Then, we compute the PSSN after removing the first 15 Zernike modes (low-order error). The von Karman PSD has three different parameters in its model defined in Fig. $\underline{6}$. They are PSD amplitude $(A)$, PSD knee frequency $\left(f_{o}\right)$, and PSD slope $(\gamma)$. We vary those parameters to find their dependency on PSSN. We compute $\alpha_{v k}$, that is, the $\alpha$ value for the von Karman PSD surface, for each parameter set after computing PSSN and the residual rmsSRF after removing the $15 \mathrm{LOZ}$ :

$$
\alpha_{v k}=1-\frac{\widehat{P S S N}}{r m s S R F_{v k}^{2}},
$$

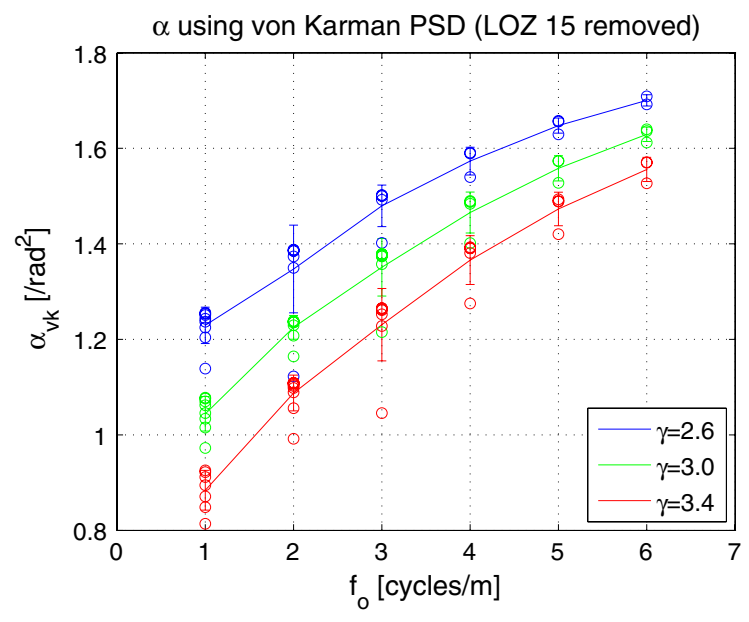

Fig. 7. Alpha values computed from Fig. $\underline{6}$ as a function of $f_{o}$ and $\gamma$ of the von Karman PSD. Since the amplitude parameter $A$ has negligible dependency, we merge its dependency as the dots on the plot.

where $\widehat{P S S N}$ and $r m s S R F_{v k}$ are the average PSSN and the average rmsSRF of the segment surfaces based on the von Karman PSD after the $15 \mathrm{LOZ}$ are removed.

Figure 7 shows the result of the study presenting $\alpha$ value dependency on $f_{o}$ and $\gamma$. Since $A$ has negligible dependency, we merge its dependency as the dots on the plot. For the evaluation, we only consider cases when the PSSN is larger than 0.98, which is our target performance according to the TMT PSSN budget for the polishing error term [3]. As shown in Fig. 7, the $\alpha_{v k}$ depends on the PSD shapes. As discussed, we choose $1.225 / \mathrm{rad}^{2}$ for $\alpha_{H O}$ for TMT, based on the parameters of $A=1.5 \mathrm{~nm}^{2} \mathrm{~m}^{2}, f_{o}=2$ cycles $/ \mathrm{m}$, and $\gamma=3$, which is a good representation of the real mirror sample group.

We also repeat this process to evaluate $\alpha_{H O}$, varying the number of LOZs. The result is tabulated in Table 3. The $\alpha_{H O}$ value increases as the number of the LOZs increases since a relatively higherfrequency spectrum is expected as more Zernikes are removed beforehand.

In order to verify these $\alpha_{H O}$, we perform a study using the real sample mirrors described in Fig. $\underline{8}$. We numerically synthesize 492 segments for a TMT M1 set using the computed PSD of the real sample mirrors shown in Fig. 5. Then, we remove the LOZ for all segments and compute rmsSRF and PSSN. The computed PSSN is compared to the approximated PSSN, namely $1-\alpha_{H O} \cdot r m s S R F^{2}$.

Figure 9 shows the result for various numbers of

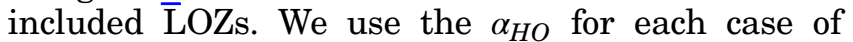
LOZ from Table $\underline{3}$.

Table 3. Various Alpha Values Corresponding to the Residual High-Order Wavefront Errors After Removing Different Numbers of LOZ Shapes from the von Karman PSD with $f_{o}=2 c / \mathrm{m}$ and $\gamma=3$

\begin{tabular}{lcccccc}
\hline No. of LOZ & 10 & 15 & 21 & 28 & 36 & 45 \\
\hline$\alpha_{H O}\left(/ \mathrm{radian}^{2}\right)$ & 1.162 & 1.225 & 1.291 & 1.355 & 1.415 & 1.473 \\
$\alpha_{H O}\left(/ \mathrm{nm}^{2}\right)$ & $7.341 \mathrm{e}-04$ & $7.738 \mathrm{e}-04$ & $8.155 \mathrm{e}-04$ & $8.556 \mathrm{e}-04$ & $8.938 \mathrm{e}-04$ & $9.304 \mathrm{e}-04$ \\
\hline
\end{tabular}




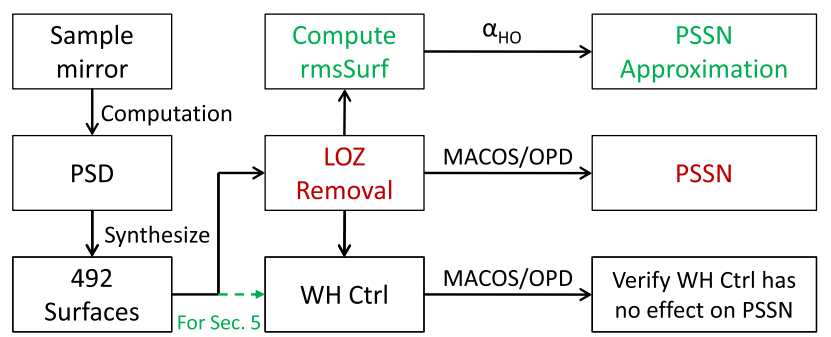

Fig. 8. Numerical validation setup using real sample mirrors for $\alpha_{H O}$ evaluated in Table 3 . We numerically synthesize 492 segments for TMT M1 set using the computed PSD of the real sample mirrors shown in Fig. 5. Then, we remove the LOZ for all segments and compute rmsSRF and PSSN. This computed PSSN is compared to the approximated PSSN, that is, $1-\alpha_{H O} \cdot r m s S R F^{2}$. In order to verify that the number of included LOZs is enough, we perform the WH correction on segments and evaluate the PSSN afterward.

In order to capture the WH controllability, we performed the following analysis. We apply the WH correction to segments and evaluate the PSSN after LOZ removal as described in the bottom part of Fig. 8 . We considered the 10 SVD modes for the WH correction. The number of LOZs removed should be large enough to make the effect of further WH correction negligible. In other words, the high-order residual wavefront error after LOZ removal should not be affected by WH shape corrections. Those residuals should fall beyond the spatial frequency bandwidth of WH control. Figure 9(c) shows that the WH correction has no impact on the high residual surfaces in terms of PSSN if LOZs of 15 or more are removed. The PSSN differences below $10^{-4}$ is difficult to resolve due to the numerical precision of PSSN computation [2].

Including more Zernikes makes the overall PSSN approximation better as shown in Fig. 9(b). However, it makes the vendor table more complicated. We chose 15 Zernikes for TMT based on Fig. 9(b), which provides a reasonable PSSN estimation accuracy while maintaining a small number of Zernikes.

\section{Surface Roughness}

With regard to PSSN, the spatial frequency aberration beyond a certain frequency can be assumed to be a white noise since all the energy scattered by those high-frequency aberrations is located beyond the atmosphere aberration boundary. According to the $\beta$ function [8], the spatial frequency aberration beyond 20 cycles/m can be assumed as a white noise for the $r_{o}$ of $200 \mathrm{~mm}$ and the wavelength of $500 \mathrm{~nm}$.

Since the spatial frequency for the surface roughness error is larger than $1 / 100 \mu \mathrm{m}^{-1}$ (or $1 \times$ $10^{4}$ cycles $/ \mathrm{m}$ ) for TMT, they can be assumed to be a white noise. Therefore, we use the $\alpha_{S R}$ value of $2 / \mathrm{rad}^{2}$ or $2 \times(4 \pi / 500)^{2} / \mathrm{nm}^{2}$, which is the maximum possible value for the $\alpha$ value [6] .

\section{Validation of Vendor Table}

In order to validate the vendor table, we conducted the following tests using the vendor table shown in Table 1.

First, we choose five different samples from three different mirror polishing vendors in Table 2. They are Vendor-B, Vendor-C, Vendor-E, Vendor-E.1, and Vendor-E.4. We exclude mirror samples whose surface qualities are not close to our TMT M1 requirement or whose physical dimensions are too large or too small compared to the TMT segment size of $1.44 \mathrm{~m}$.

Next, we obtain MUTs for the TMT segment after truncating (cookie-cutting) the selected samples into the TMT M1 segment shape. Since the dimensions of the samples are smaller than the TMT's $1.44 \mathrm{~m}$ hexagon, we spatially stretch the mirrors to just meet the $1.44 \mathrm{~m}$. This spatial stretching effectively improves the surface quality. However, we assume this effect is negligible since the stretching ratio is small (2.5\% for Vendor-E, E.1, and E.2, and 3.9\% for other segments).

We apply the TMT vendor table to each MUT to obtain the vendor table estimated PSSN. This PSSN estimated by the vendor table is compared to three different cases of PSSN estimation.

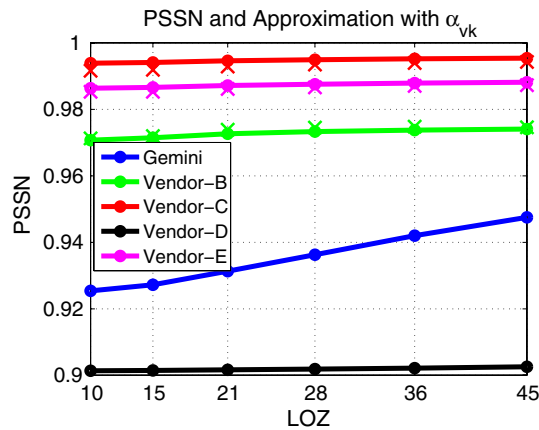

(a)

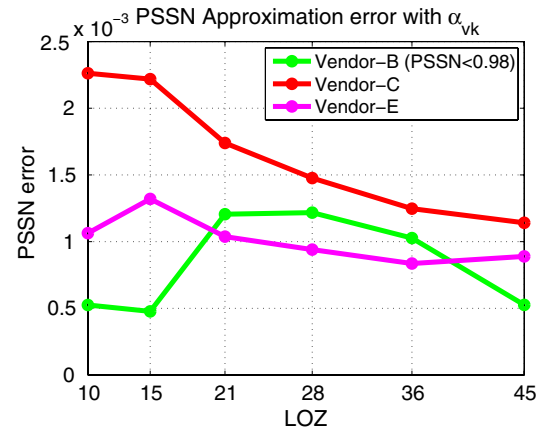

(b)

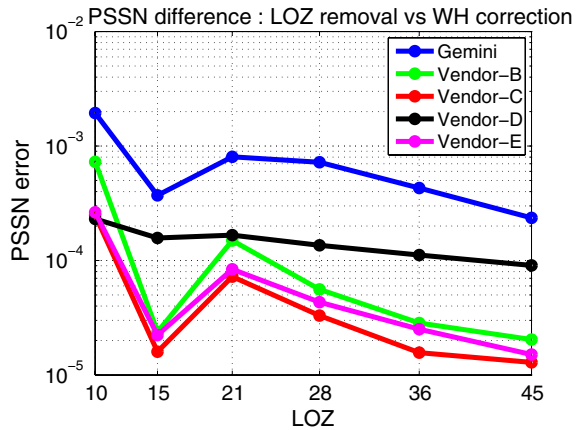

(c)

Fig. 9. (a) Actual PSSN for mirror samples after LOZ removal. For the samples whose PSSNs are larger than 0.96, their approximated PSSNs using $\alpha_{v k}$ are also marked with the same colored " $\times$." (b) PSSN difference (Actual PSSN-Approximated PSSN) for sample mirrors that generate PSSN higher than 0.96. (c) PSSN difference for mirror samples after LOZ removal and after WH correction as described in the bottom part of Fig. $\underline{8}$. 


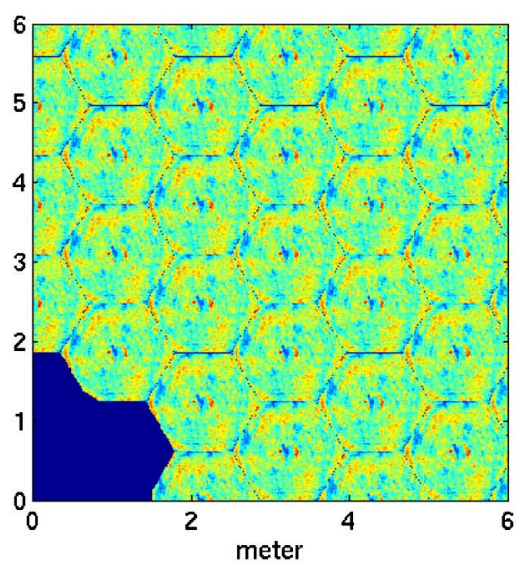

(a) Replicated I

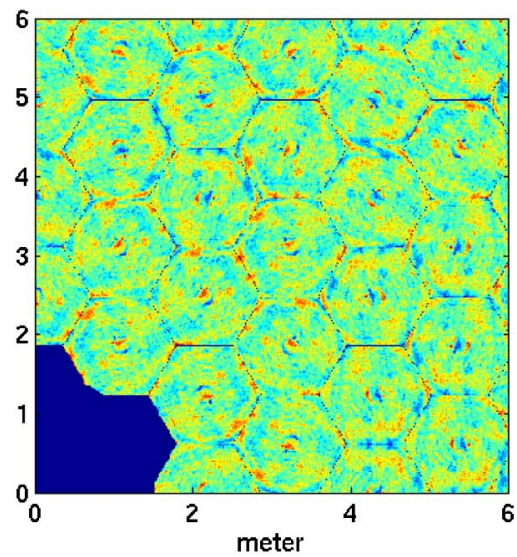

(b) Replicated II

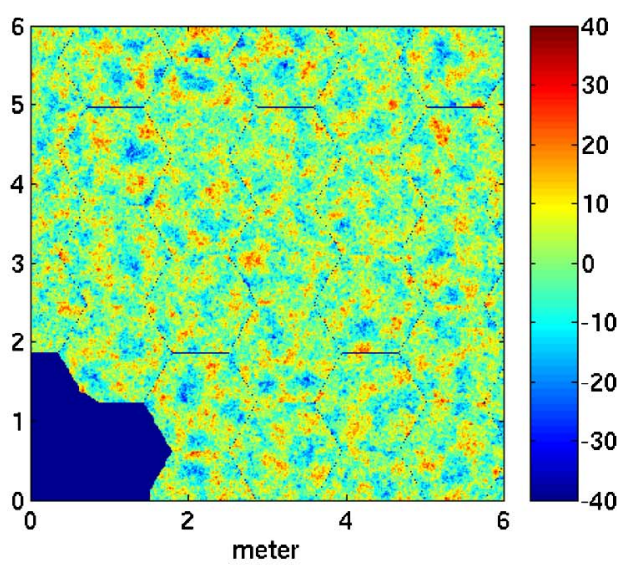

(c) PSD-Based

Fig. 10. Differently configured telescopes are compared conceptually. (a) Replicated I: we replicate the MUTs and install them homogeneously in one direction for all 492 segments. We remove the piston, tip, and tilt and apply the 10 SVD WH correction to all segments. (b) Replicated II: similarly as with the "Replicated I" case, we replicate the MUT. However, we orient the segments randomly by rotating segments, that is, by $60 \mathrm{deg}, 120 \mathrm{deg}$, and so forth. We also randomly flip the segment surfaces numerically by changing the sign. (c) PSDbased method: we numerically synthesize the 492 segments using the PSD computed from the MUT. All segments have the same (or same within the numerical precision) PSD as the MUT, but the phase information of the MUT is lost or random from segment to segment. This procedure is depicted in Fig. $\underline{8}$ as indicated by "For Section $\underline{5}$. "

1. Replicated I: we numerically replicate the MUTs and install them homogeneously in one direction for all 492 segments as shown in Fig. 10(a). We remove the piston, tip, and tilt and apply the 10 SVD $\mathrm{WH}$ correction to all segments. Then, we compute the OPD and the PSSN, using our optical-ray tracing software, MACOS. The computed PSSN and rmsWFE are shown in Table $\underline{4}$ under "Replicated I."

2. Replicated II: Similarly as with the "Replicated I" case, we replicate the MUT. However, we orient the segments randomly by rotating segments, that is, $60 \mathrm{deg}, 120 \mathrm{deg}$, and so forth. We also randomly flip the segment surfaces numerically by changing the sign. The generated OPD from MACOS is shown in Fig. 10(b).

3. PSD-based Method: We numerically synthesize the 492 segments using the PSD computed from the MUT. All segments have the same (or same within the numerical precision) PSD as the MUT, but the phase information of the MUT is random from segment to segment. This procedure is depicted in Fig. $\underline{8}$ as indicated "For Section $\underline{5}$.

The two replicated cases, 1 and 2, represent the two most homogeneous (coherent) cases as discussed in Section $\underline{3}$, having only considered random orientation of segment installation. They both assume that the surfaces errors of the MUT are deterministic errors. On the other hand, the PSD-based method assumes the surface errors of the MUT are all random errors. Therefore, the two replicated methods and the PSD-based method represent two extreme cases of telescope performance based on the MUT input.

For the test, we make additional assumptions as described below. The TMT WH is considered for the surface correction using an ideal ShackHartmann wavefront sensor (SH-WFS) with five subaperture rings on the segment [11]. By an ideal SH-WFS, we mean that subaperture slopes (tip/tilt)

Table 4. Validation of Vendor Table: The Computed or Estimated PSSN and rmsWFE Values for Various Methods in Section $\mathbf{5}$ are Listed under Each Corresponding Column ${ }^{a}$

\begin{tabular}{|c|c|c|c|c|c|c|c|c|}
\hline Mirror & \multicolumn{2}{|c|}{ Replicated I } & \multicolumn{2}{|c|}{ Replicated II } & \multicolumn{2}{|c|}{ Vendor Table } & \multicolumn{2}{|c|}{ PSD-based Method } \\
\hline Vendor-B & $\begin{array}{c}0.974026 \\
(1.57 \mathrm{e}-03)\end{array}$ & $\begin{array}{l}10.6 \\
(-1.6)\end{array}$ & $\begin{array}{c}0.973528 \\
(1.07 \mathrm{e}-03)\end{array}$ & $\begin{array}{l}10.6 \\
(-1.6)\end{array}$ & $\begin{array}{c}0.972455 \\
\quad(0.0)\end{array}$ & $\begin{array}{l}12.2 \\
(0.0)\end{array}$ & $\begin{array}{c}0.970693 \\
(-1.76 e-03)\end{array}$ & $\begin{array}{l}11.9 \\
(-0.4)\end{array}$ \\
\hline Vendor-C & $\begin{array}{c}0.997032 \\
(3.64 \mathrm{e}-03)\end{array}$ & $\begin{array}{c}5.1 \\
(-0.9)\end{array}$ & $\begin{array}{c}0.996520 \\
(3.13 \mathrm{e}-03)\end{array}$ & $\begin{array}{c}5.1 \\
(-0.9)\end{array}$ & $\begin{array}{c}0.993393 \\
(0.0)\end{array}$ & $\begin{array}{l}6.0 \\
(0.0)\end{array}$ & $\begin{array}{c}0.993876 \\
(4.83 \mathrm{e}-04)\end{array}$ & $\begin{array}{l}6.5 \\
(0.5)\end{array}$ \\
\hline Vendor-E & $\begin{array}{c}0.988982 \\
(2.57 \mathrm{e}-03)\end{array}$ & $\begin{array}{c}7.2 \\
(-1.9)\end{array}$ & $\begin{array}{c}0.988805 \\
(2.39 \mathrm{e}-03)\end{array}$ & $\begin{array}{c}7.2 \\
(-1.9)\end{array}$ & $\begin{array}{c}0.986414 \\
\quad(0.0)\end{array}$ & $\begin{array}{c}9.1 \\
(0.0)\end{array}$ & $\begin{array}{c}0.986369 \\
(-4.50 \mathrm{e}-05)\end{array}$ & $\begin{array}{c}8.4 \\
(-0.7)\end{array}$ \\
\hline Vendor-E.4 & $\begin{array}{c}0.918526 \\
(3.52 \mathrm{e}-02)\end{array}$ & $\begin{array}{c}24.3 \\
(-2.3)\end{array}$ & $\begin{array}{c}0.919744 \\
(3.64 \mathrm{e}-02)\end{array}$ & $\begin{array}{c}24.3 \\
(-2.3)\end{array}$ & $\begin{array}{c}0.883353 \\
(0.0)\end{array}$ & $\begin{array}{l}26.6 \\
(0.0)\end{array}$ & $\begin{array}{c}\text { N/A } \\
\text { (N/A) }\end{array}$ & $\begin{array}{l}\text { N/A } \\
\text { (N/A) }\end{array}$ \\
\hline
\end{tabular}

${ }^{a}$ The values in parentheses are the difference values to the values using the vendor table. 
are determined numerically, not using centroiding of the PSF of each subaperture. When applying WH, we consider the 10 SVD modes for the surface correction as it is the baseline scheme [11]. All segments are assumed to be the same with the same optical aperture on top of M1 surface. No surface roughness error or lower-order allowances are considered. Surfaces and segments are sampled by interpolation with $1 / 64 \mathrm{~m}$ resolution when we obtain the OPD at the exit pupil. We also consider the one-dimensional (1D) PSD after azimuthally averaging the 2D PSD, thus assuming also that the surfaces are isotropic.

Table 4 summarizes the study results, where the compute $\bar{d}$ and estimated PSSN and rmsWFE values are listed under each corresponding column. The values in the parenthesis are the difference values to the values using the vendor table. Figure 11 also plots the PSSN values where the Vendor-E. $4 \overline{\mathrm{PSSN}}$ values are located outside the plotting range.

The study results can lead to important conclusions. As a major observation, the PSSN values from the vendor table are worse than those from the two replicated cases and are close to the PSD-based method for Vendor-C and Vendor-E. Note that the PSD-based method assumes the extreme scenario, which is supposed to generate the worst PSSN as discussed. This demonstrates that the PSSN estimation using the vendor table approximates the worst-case PSSN at least for Vendor-C and Vendor-E.

However, we observe discrepancy between the two PSSN values of the vendor table and the PSD-based method for mirror samples of Vendor-B and VendorE.1. Since the vendor table is conceptually based on the PSD method, both PSSN estimations are expected to generate the worst PSSN. We find the two following situations to make those PSSN estimations differ from the worst PSSN.

The PSSN estimation using the vendor table can be different from the worst PSSN if the MUT fails to meet our expectation. The vendor table assumes that the MUT has a certain expected behavior such as the PSD slope and PSD frequency knee. For the

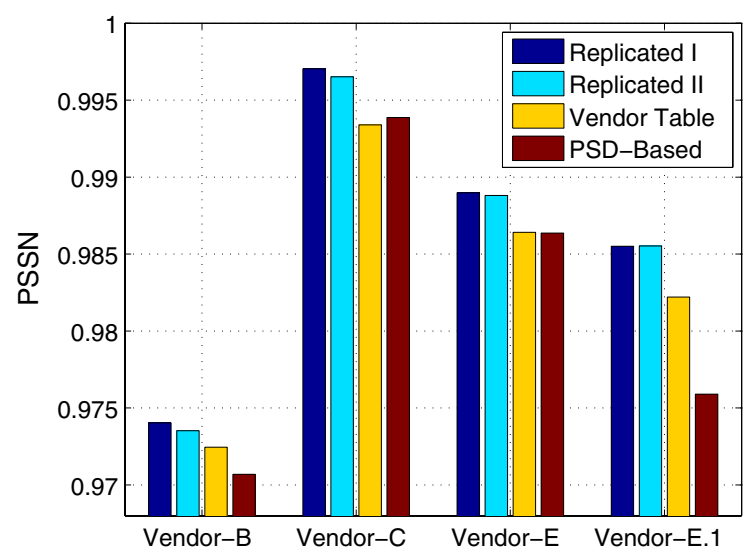

Fig. 11. Validation of vendor table: the computed or estimated PSSN values are shown for various methods described in Section 5 . Their numerical values are also listed in Table $\underline{4}$.
TMT vendor table, we explore several existing mirror samples in Fig. $\underline{5}$ and conclude that the future target sample mirror would have the PSD slope and frequency knee close to 3 and 2 cycles/m, respectively. If they are too different from this expectation, the $\alpha_{H O}$ coefficient needs to be reevaluated and the vendor table updated accordingly. Therefore, care should be taken when using and designing a vendor table so that the PSSN estimation using the vendor table is defined to have the worst PSSN.

The PSSN estimation using the PSD method can be different from the worst PSSN if the MUT fails to satisfy the condition where the PSD computation is valid. Suppose $S$ is the $2 \mathrm{D}$ surface and $F(S)$ is its Fourier transform. In the strict sense, its PSD is defined as either the Fourier transform of the autocorrelation function of $S$ or $|F(S)|^{2}$ if the two results are identical. This condition is analogous to that in which $S$ has shift-invariant or homogeneous characteristics. If a MUT has a strong unique deterministic component, such as spiral or circular grooves, straight stripes, or poor edges, the PSD obtained from the PSD computation algorithm becomes erratic. Unlike other mirror samples, Vendor-B shows a strong low frequency and Vendor-E.1 has bad edges, introducing a fictitious energy increase in the high-frequency regime as shown in Fig. 5. Therefore, care should be also taken when applying the PSD method.

As a minor observation of comparing the two replicated cases, the PSSN values of "Replicated I" are higher than those of "Replicated II" except for Vendor-E.1 and Vendor-E.4. This can be expected, since the "Replicated I" case is a more coherent case than "Replicated II." However, the reason for the two exceptions (Vendor-E.1 and Vendor-E.4) is not clear. One hypothesis is that the edge discontinuities between segments in the "Replicated II" case are larger, causing additional energy spreading into the higher spatial frequency band.

\section{Summary}

The properly designed vendor table estimates the worst-case PSSN for all possible realizations of a segmented telescope when it consists of segments whose PSD is the same as an input MUT. While the vendor table considers all of the complex natures of the telescope's operation and controls such as piston, tip/tilt control, and surface corrections via WH, its PSSN estimation process is made simple and straightforward by shielding the details from the vendor. Therefore, it will be useful for the individual segment specifications such as the segment polishing specification. Although we introduce a vendor table specifically built for the TMT's segment polishing specification, the concept of the vendor table will be applicable to other segmented telescopes as well.

This research was carried out in part at the Jet Propulsion Laboratory, California Institute of Technology, and was sponsored by the California Institute of Technology and the National Aeronautics and Space Administration. The authors gratefully 
acknowledge the support of the TMT partner institutions. They are the Association of Canadian Universities for Research in Astronomy (ACURA), the California Institute of Technology, and the University of California. This work was supported as well by the Gordon and Betty Moore Foundation, the Canada Foundation for Innovation, the Ontario Ministry of Research and Innovation, the National Research Council of Canada, the Natural Sciences and Engineering Research Council of Canada, the British Columbia Knowledge Development Fund, the Association of Universities for Research in Astronomy (AURA), and the U.S. National Science Foundation.

\section{References}

1. G. Z. Angeli, S. Roberts, and K. Vogiatzis, "Systems engineering for the preliminary design of the Thirty Meter Telescope," Proc. SPIE 7017, 701704 (2008).

2. C. Nissly, B. Seo, M. Troy, G. Angeli, J. Angione, I. Crossfield, B. Ellerbroek, L. Gilles, N. Sigrist, and L. Wang, "Highresolution optical modeling of the Thirty Meter Telescope for systematic performance trades," Proc. SPIE 7017, 70170U (2008).

3. G. Angeli, "Seeing limited PSS budget D16v2," TMT Project Communication TMT.SEN.DRD.07.026.REL16 (2012).
4. H. Bonnet, M. Esselborn, M. Muller, B. Sedghi, and F. Koch, "E-ELT active optics system modeling and performance evaluation," Proc. SPIE 8336, 83360P (2011).

5. I. R. King, "Accuracy of measurement of star images on a pixel array," Publ. Astron. Soc. Pac. 95, 163-168 (1983).

6. B.-J. Seo, C. Nissly, G. Angeli, B. Ellerbroek, J. Nelson, N. Sigrist, and M. Troy, "Analysis of normalized point source sensitivity as performance metric for large telescopes," Appl. Opt. 48, 5997-6007 (2009).

7. B.-J. Seo, C. Nissly, M. Troy, G. Angeli, R. Bernier, L. Stepp, and E. Williams, "TMT M1 segment polishing specification analysis (draft)," TMT Project Communication TMT.SEN. TEC.11.018.DRF01 (2011).

8. B.-J. Seo, C. Nissly, M. Troy, G. Angeli, V. Ford, L. Stepp, and E. Williams, "Approximation of normalized point source sensitivity using power spectral density and slopes of wavefront aberration" Appl. Opt. 17, 3910-3922 (2013).

9. R. J. Noll, "Zernike polynomials and atmospheric turbulence," J. Opt. Soc. Am. 66, 207-211 (1976).

10. Thirty Meter Telescope, "Specification for finished 1.44-meter primary mirror segments," TMT Project Communication TMT.OPT.SPE.07.002.CCR03 (2008), http://www.tmt.org/sites/ default/files/TMT-OPT-SPE-07-002-CCR03-Specification-forFinished-Primary-Mirror-Segments-Final.pdf.

11. B. Seo, C. Nissly, G. Angeli, D. MacMynowski, N. Sigrist, M. Troy, and E. Williams, "Investigation of primary mirror segment's residual errors for the Thirty Meter Telescope," Proc. SPIE 7427, 74270F (2009).

12. B.-J. Seo, C. Nissly, M. Troy, and G. Angeli, "TMT figuring error modeling," TMT Project Communication TMT.SEN TEC.10.025.DRF01 (2010). 